Muséologies

Les cahiers d'études supérieures

muséologies

\title{
Palladio à l'oeuvre, Architecture en uniforme et les nouveaux programmes en muséologie au Centre Canadien d'Architecture
}

\section{Alessandra Mariani}

Volume 5, numéro 2, printemps 2011

URI : https://id.erudit.org/iderudit/1033519ar

DOI : https://doi.org/10.7202/1033519ar

Aller au sommaire du numéro

Éditeur(s)

Association Québécoise de Promotion des Recherches Étudiantes en

Muséologie (AQPREM)

ISSN

1718-5181 (imprimé)

1929-7815 (numérique)

Découvrir la revue

Citer ce compte rendu

Mariani, A. (2011). Compte rendu de [Palladio à l'oeuvre, Architecture en uniforme et les nouveaux programmes en muséologie au Centre Canadien

d'Architecture]. Muséologies, 5(2), 164-175. https://doi.org/10.7202/1033519ar d'utilisation que vous pouvez consulter en ligne. 
Alessandra Mariani

Palladio à l'œuvre, Architecture en uniforme et les nouveaux programmes en muséologie au Centre Canadien d'Architecture 


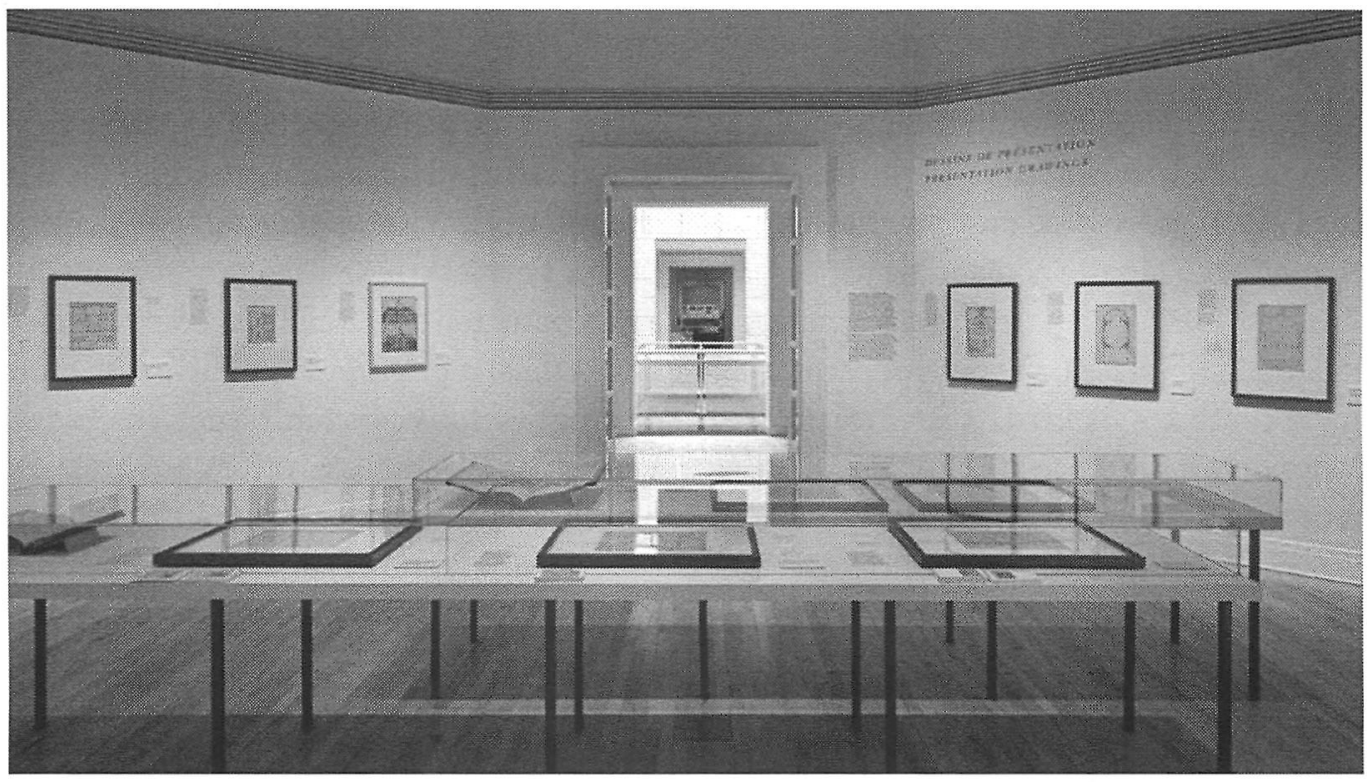

Vue de l'exposition du CCA,

Palladio à l'ceuvre. @ CCA, Montréal

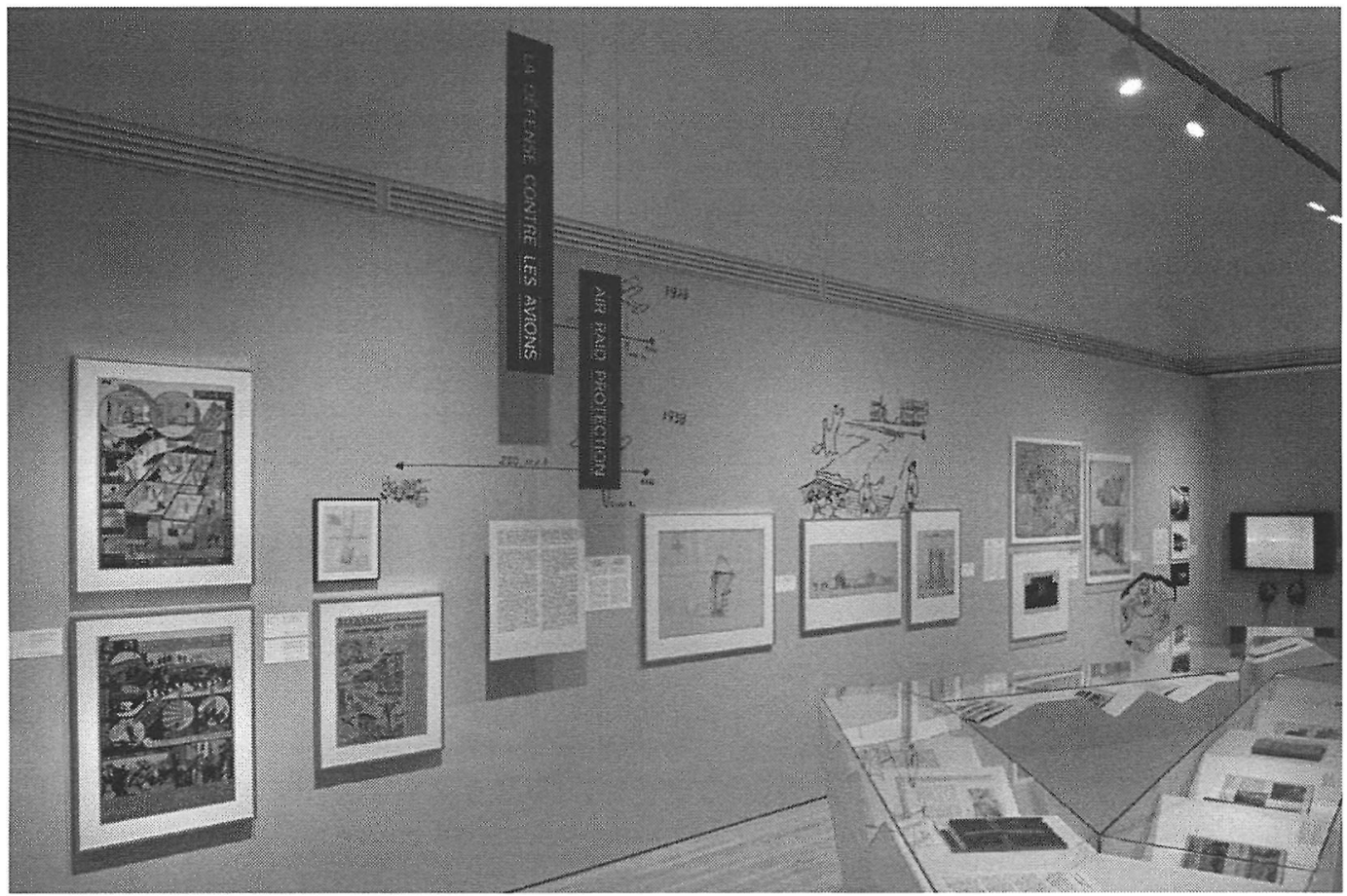

Vue l'exposition du CCA, Architecture en uniforme: projeter et construire pour la Seconde Guerre mondiale . Centre Canadien d'Architecture, Montréal. 
Le Centre Canadien d'Architecture (CCA) a inauguré ce printemps deux expositions qui transforment la formule habituelle de l'exposition historique. La première, Palladio à l'cuvre', présentée du 3 mars au 22 mai dernier dans la salle octogonale du Centre, réunissait une quinzaine de dessins d'Andrea Palladio provenant des collections du Royal Institute of British Architects, des documents extraits de la collection du CCA, dont une version originale datée de 1570 de son traité I Quattro Libri dell'Architettura ${ }^{2}$ et un dessin de l'un de ses élèves, vincenzo Scamozzi. Cuido Beltramini, commissaire de l'exposition, a cherché à exposer la progression et la transformation dans le temps des méthodes de travail de Palladio par l'assemblage de gravures et de textes qui imageaient le parcours de l'architecte. Né en 1508, Andrea Palladio reçut à l'adolescence une formation de dessinateur et de tailleur de pierre. Il construisit des églises à Venise ainsi que des villas et des palais à Vicence et dans la Vénétie. L'architecture antique imprégna autant ses dessins que ses constructions: il en vint à écrire I Quattro Libri parce qu'il souhaitait résoudre les divergences entre les modes de construction usuels de l'époque et les constructions plus nettement achevées de l'Antiquité décrites par ses prédécesseurs, Vitruve et Alberti.

Voir <http://www.cca.qc.ca/fr/expositions/1205-palladio-a-l-oeuvre> (consulté le 25 mai 2011).

2

I Quattro Libri dell'Architettura [Les quatre livres d'architecture] est un ouvrage composé de quatre sections ou "livres" où sont colligés des projets architecturaux que Palladio retient exemplaires et qu'il documente et illustre abondamment par des coupes, des plans, des élévations et des éléments d'architecture.
La mise en exposition de Palladio à l'ceuvre - évocatrice du cabinet de curiosités aurait pu, de prime abord, n'appeler que le connaisseur, mais la charge esthétique des documents et la séquence de leur présentation permettaient une compréhension aisée pour tout visiteur. Le commissaire avait choisi d'orienter le public par le biais de cinq sections concomitantes au cheminement de l'architecte, favorisant ainsi un parcours didactique. La première présentait trois dessins de ruines antiques réalisés par Palladio, la copie de monuments de l'Antiquité étant un rite, un passage obligé et formateur pour de nombreux artistes et architectes de cette période. La deuxième section montrait deux esquisses rares qui captaient un moment d'idéation; les dessins d'étude de la troisième section traduisaient l'étape de la conception où l'idée est instrumentalisée, où la mise en forme est produite à partir de limites réelles. Venaient ensuite un regroupement de dessins plus léchés et graphiquement investis d'effets facilitant la communication de ses idées. Des livres d'architecture fermaient cette boucle, confirmant l'apport substantiel de Palladio au développement de la représentation architecturale: parmi ceux-ci, son ouvrage I Quattro Libri dell'Architettura, un modèle pour la transmission des idées en architecture.

La seconde exposition, Architecture en uniforme ${ }^{3}$, présentée du 13 avril au 18 septembre 2011, est la mise en forme d'un nouveau récit qui retrace la contribution des architectes au cours de la Seconde Guerre Voir <http://www.cca.qc.ca/fr/expositions/1ו12-architecture-en-uniforme> (consulté le 25 mai 2011). 


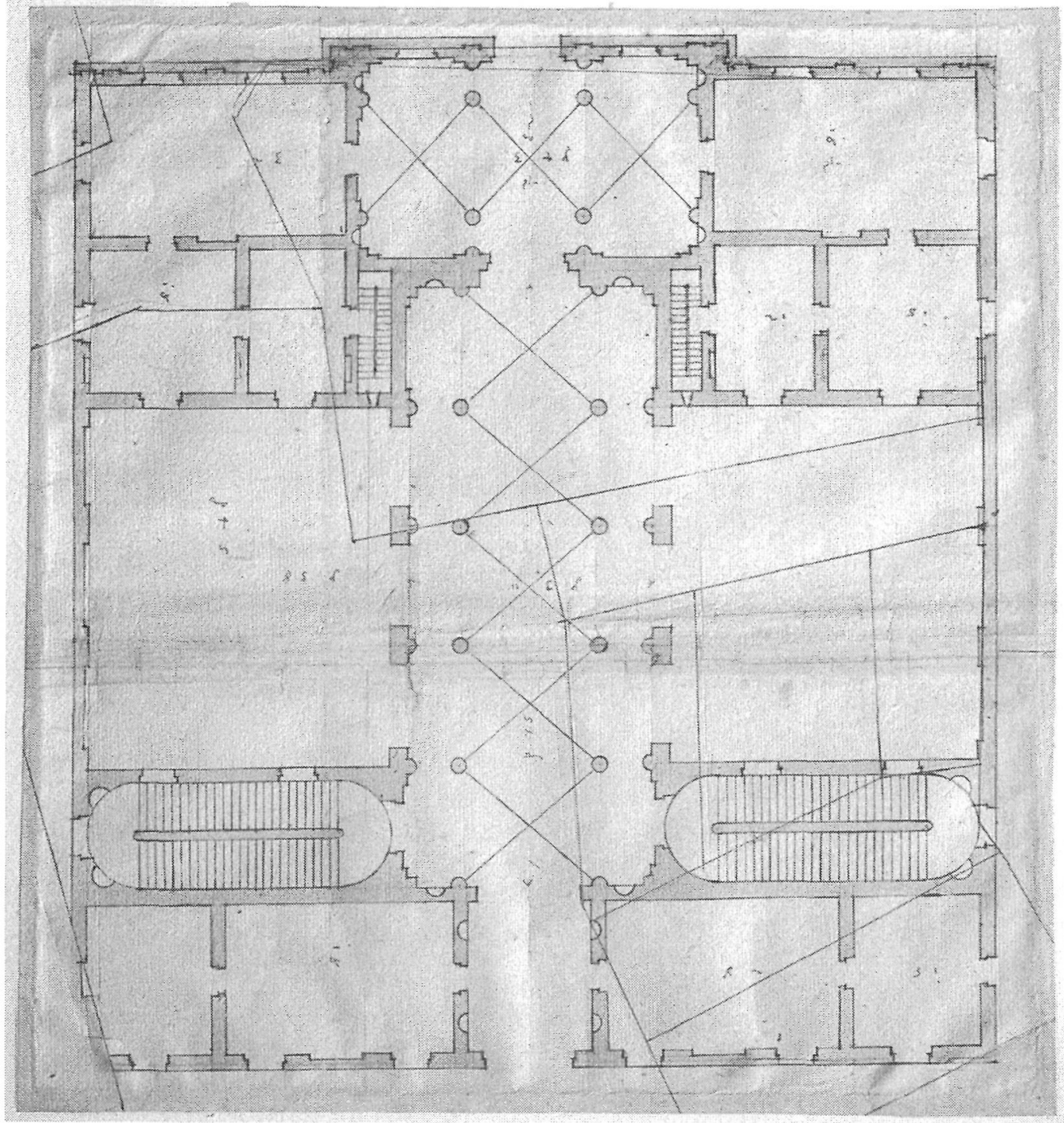

Andrea Palladio. Étude d'un plan de palais à deux cours. Vers 1570 . Londres, Bibliothèque du Royal Institute of British Architects.

$\mathrm{XVI} /$ 1or 

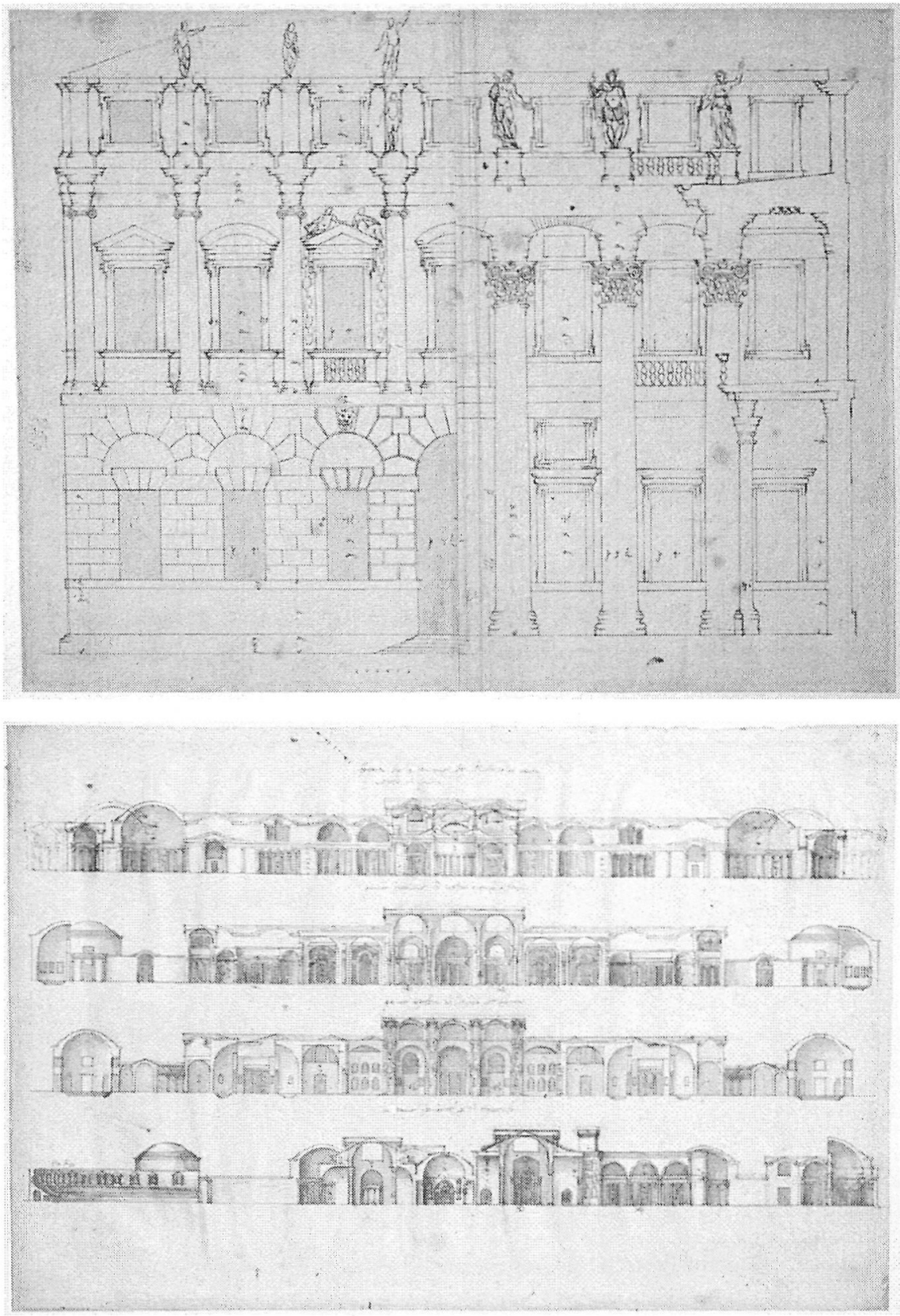

(Illustration du haut)

Andrea Palladio. Études pour les planches du palais de Porto parues dans I Quattro Libri : façade et section correspondant à la cour d'ordre colossal. Vers 1560. Londres, Bibliothèque du Royal Institute of British Architects. XVII/3I
(Illustration du bas)

Andrea Palladio. Étude pour une planche des thermes de Dioclétien destinée à un ouvrage portant sur "les thermes romains": coupe transversale et coupe longitudinale. Après environ 1570.Londres, Bibliothèque du Royal Institute of British Architects. V/2r 
mondiale. En souhaitant combler une période de l'histoire de l'architecture passée sous silence, le commissaire de l'exposition, Jean-Louis Cohen, lève le voile tant sur les origines d'un bon nombre de découvertes techniques, matérielles et systémiques que sur les opérations de mobilisation et de réaffectation des architectes pendant cette période. Du bombardement nazi à Guernica en 1937 à l'atomisation d'Hiroshima et de Nagasaki par les Américains en 1945, Architecture en uniforme documente, sans s'enliser dans un discours historique opératoire, le travail des architectes et la transformation de la pratique architecturale. Plus de 300 documents sont mis à contribution pour cimenter le récit de l'exposition. Des dessins originaux, des maquettes, des photographies, des documents historiques, des affiches, des livres, des publications issus du CCA et d'institutions internationales sont unis à des extraits filmiques de l'Office national du film du Canada pour constituer les huit thèmes principaux qui structurent l'exposition: Le front domestique, Cuerre aux villes, Produire la production de guerre, Architectures mobiles, La forteresse Europe, Camoufler ou le dessin de l'invisible, Quatre macro-projets et De la guerre à la paix.

La riche matérialité de l'exposition exprime efficacement la diversité et l'ampleur du travail des architectes mené au cours de la guerre. Au sas d'entrée, deux photographies murales annoncent l'impact des événements et leurs répercussions. Un espace circulaire accueille le visiteur dans la première salle: telle une résurrection apothéotique, la galerie de portraits qu'elle recèle présente les protagonistes du récit. Dans les salles, une quantité de propos se succèdent: le dynamisme de la mise en scène est surtout focalisé sur l'éventail de l'expérimentation et des découvertes. On y apprend que la contribution des architectes à l'effort de guerre, même s'ils n'ont pas tous exercé leur profession au cours des conflits, se traduisit par des tâches pouvant être accomplies grâce à leurs habiletés conceptuelles, organisationnelles et créatives. Mobile, urbaine et aérienne, la Seconde Guerre mondiale a exigé une profonde réorganisation de la main-d'œuvre puisque le travail était essentiellement orienté sur la défense des territoires, la production d'armements et d'engins militaires, la création de systèmes préfabriqués et de bâtiments mobiles conçus pour le déploiement des troupes. L'inventivité des architectes fut mise à profit dans la recherche de matériaux nouveaux et de substitution essentiels sur les plans domestique, industriel et militaire. Deux salles de l'exposition consacrées à la construction d'industries militaires (vouées à la fabrication d'avions, de véhicules et de munitions) et à l'apparition de nouvelles villes ouvrières éloignées des grands centres urbains rendent compte aussi de la métamorphose précipitée de la cartographie de certains territoires. Parmi les cas présentés figurent les 600 unités d'habitation (aujourd'hui démolies) du quartier Channel Heights à San Pedro en Californie conçues par Richard Neutra pour les ouvriers des chantiers de la marine militaire américaine ainsi que les baraques en tôle métallique ondulée Quonset qui ont abrité soldats et réfugiés.

De nombreux dessins et documents de l'exposition renseignent sur la pratique du camouflage, application développée 


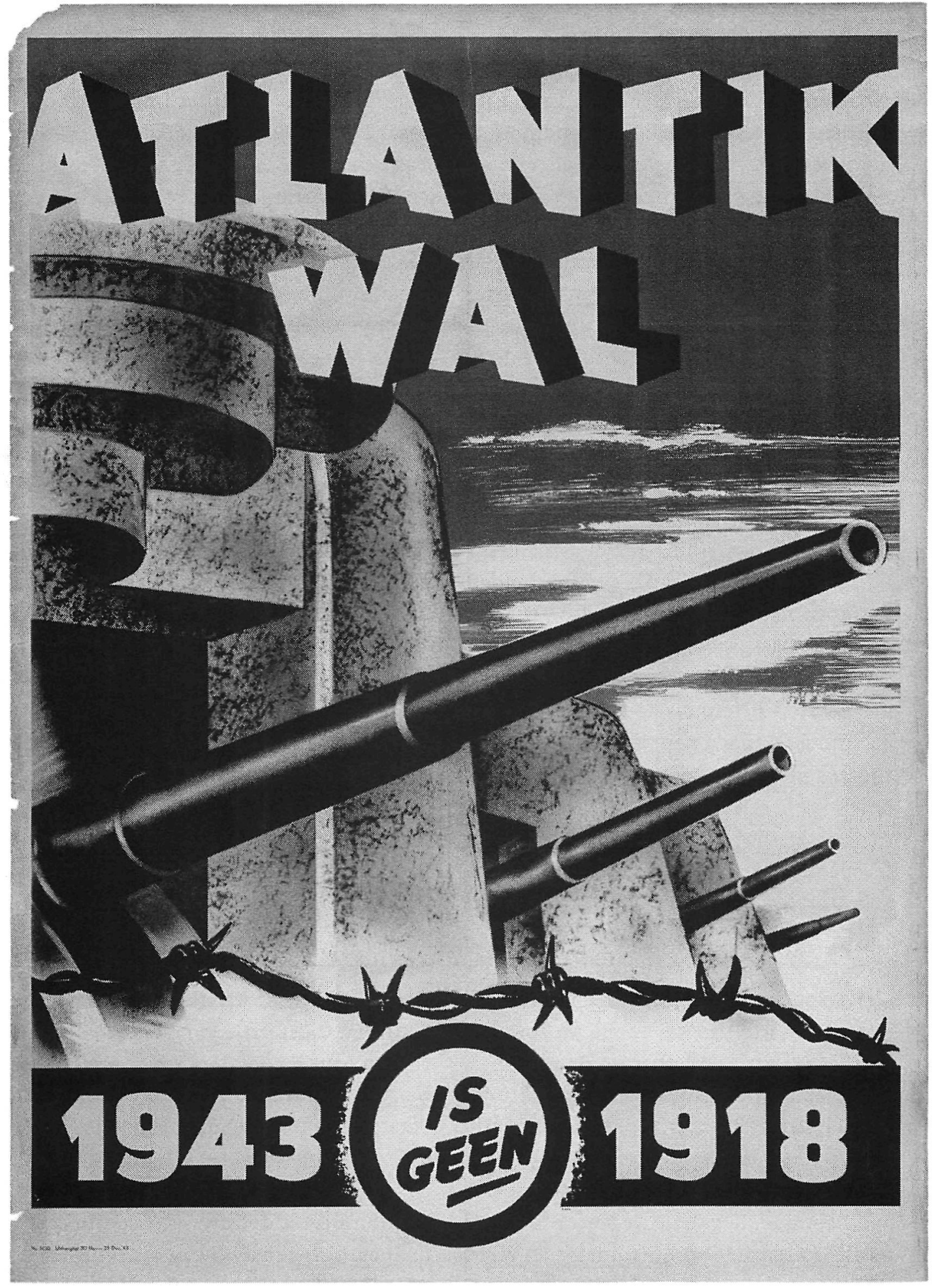




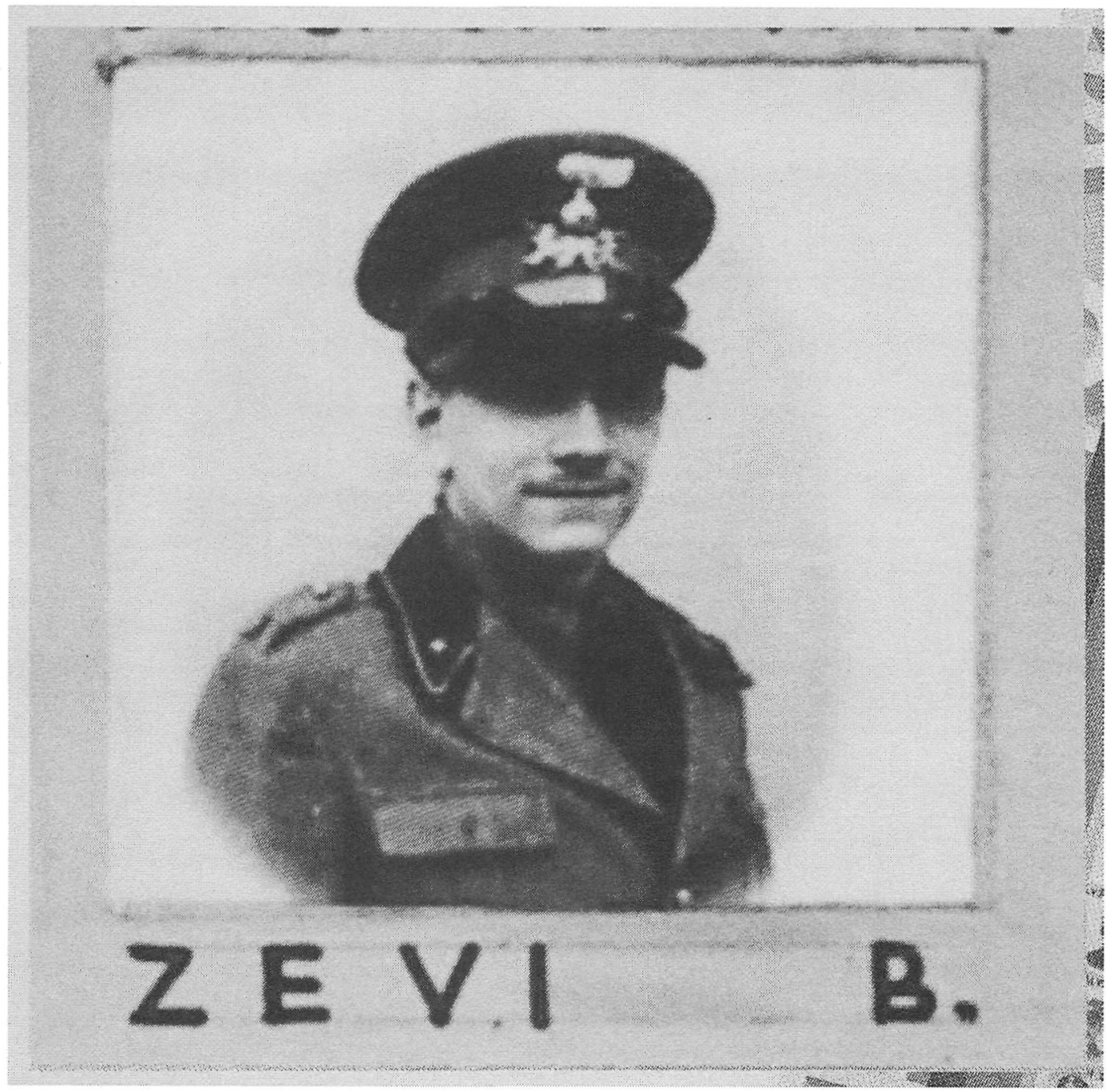

Bruno Zevi en uniforme de l'armée britannique, 1944, dans Tutto Zevi 1934-2000. Collection CCA.

"Mur de l'Atlantique; 1943 n'est pas 1918 ", affiche allemande imprimée aux Pays-Bas, 1943. The WolfsonianFlorida International University, Miami Beach, Florida, The Mitchell Wolfson, Jr. Collection, XX1990.2907 


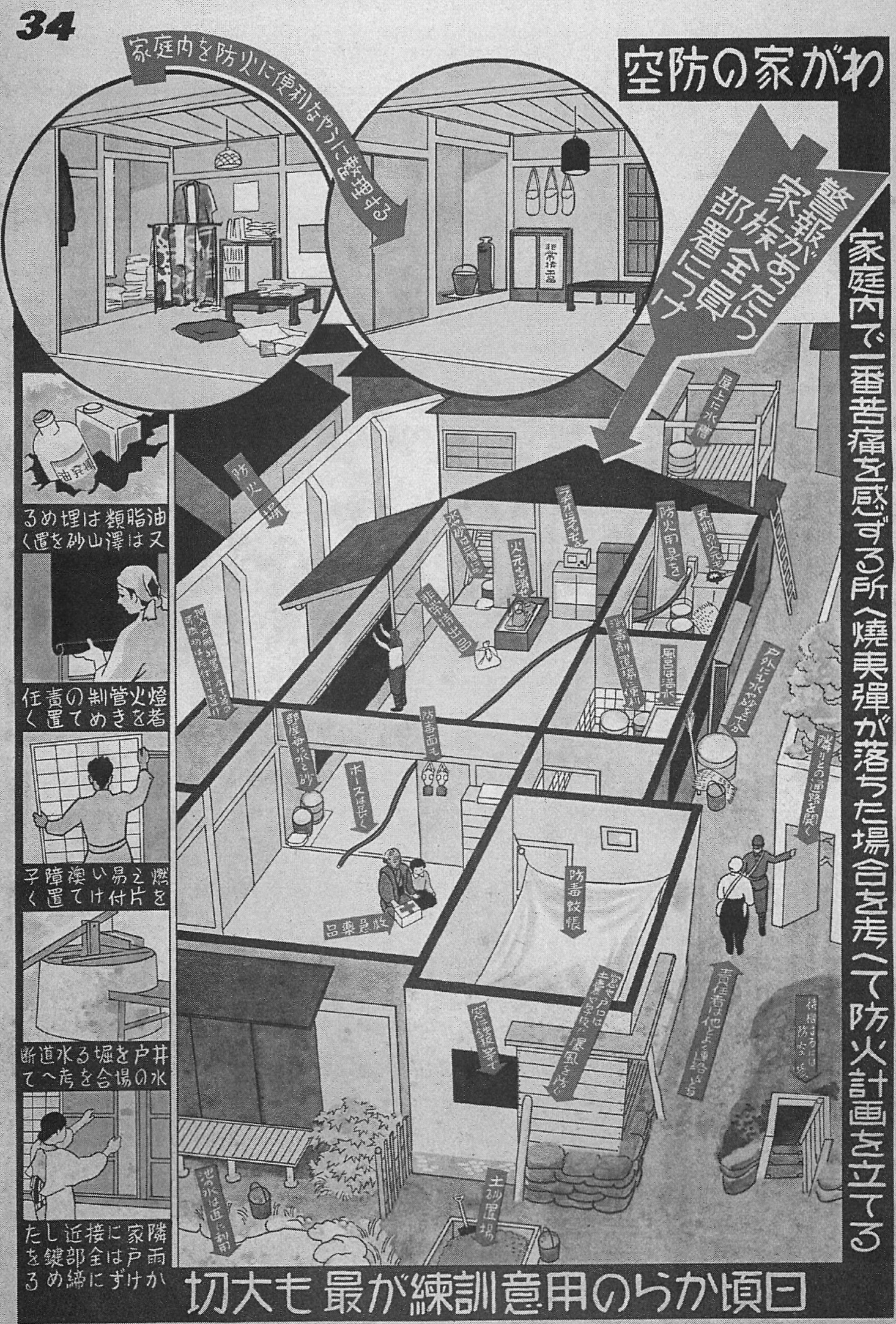


"Consignes pour la protection contre les raids aériens", Tokyo, 1943. The Wolfsonian-Florida International University, Miami Beach, Florida, The Mitchell Wolfson,

Jr. Collection, XB2002.07.26.034 
initialement par les peintres post-cubistes pour soustraire à la vue les objets au sol. Cette technique fut perfectionnée par les architectes de la Seconde Guerre pour dissimuler l'urbanisme de territoires entiers et en recréer de nouveaux, complètement inhabités, afin de se protéger des attaques aériennes. Le camouflage, "exercice de "désurbanisation" et de géographie nouvelle des territoires ${ }^{4} "$, exigeait des connaissances pointues de visualisation et de lecture du paysage. L'application de cette technique serait aussi, selon le commissaire, une première réflexion de la psychologie de la configuration (les principes de perception de la Cestalt ${ }^{5}$ ) au service de l'architecture. L'exposition suggère aussi une perception binaire de l'histoire : l'emploi du camouflage, par exemple, ne fut pas limité à une stratégie défensive. Cette exploration de l'artifice voit notamment son aboutissement dans la reproduction à l'identique d'un village japonais artificiel, en plein

4

Définition livrée par Jean-Louis Cohen lors de la visite de presse du 12 avril 2011.

5

Gestalt est un terme issu de la psychologie qui signifie un tout unifié. Ce terme réfère aux théories de la perception visuelle développées par les psychologues allemands au cours des années 1920; la Gestalt cherche à définir comment les êtres humains tendent à organiser des éléments visuels - en groupes ou en élément unifiés - lorsque certains principes (la similarité, la continuation, la complétion, la proximité) sont appliqués. Ces regroupements débouchent notamment sur des considérations de figure et de forme. Appliquées à l'architecture, par exemple, ces théories permettent de créer un certain équilibre en synchronisant l'interaction des éléments composant un bâtiment, générant ainsi un système; ou encore, elles permettent la création de tensions réelles qui deviennent un effet recherché par le concepteur. (Voir SAINT-MARTIN, Fernande. La théorie de la Gestalt et l'art visuel. Essai sur les fondements de la sémiotique visuelle. Québec: Presses de l'Université du Québec, 1990.) désert américain, construit pour tester l'efficacité du napalm. Indépendamment de leurs visées, ces pratiques fondées sur des démarches expérimentales transformeront la posture essentiellement créative et "projectuelle" de la pratique de l'architecture d'avant-guerre.

Les idéologies tayloristes et fordistes - composantes essentielles du développement des récits de l'architecture moderne de JeanLouis Cohen - prennent des dimensions considérables dans l'exposition Architecture en Uniforme. La transposition de l'énormité de la production industrielle aux projets architecturaux impose une division et une programmation du travail jusqu'alors non imaginées. La nécessité de relier les zones de production à celles d'approvisionnement et de distribution change aussi l'échelle et la nature de la conceptualisation des projets. Des quatre exemples exposés, citons-en deux: la ville industrielle d'Auschwitz où les nazis construisent une usine chimique de très grande dimension pour combler les besoins militaires est reliée au système ferroviaire et est équipée de camps de concentration où logent les ouvriers-prisonniers; la construction du Pentagone, le plus grand immeuble de bureaux réalisé en quinze mois pour le compte du ministère de la Défense américaine, transforme le projet d'architecture en projet territorial et militaire.

L'exposition se conclut sur une ouverture : la présentation de projets qui aspirent à la fin du conflit, même en temps de guerre, et qui imaginent la reconstruction en mettant à profit autant les avancées technologiques que la transformation des 
produits militaires. On y profile les débuts de la consommation de masse, dont de nombreux produits sont issus des résidus de guerre.

Si l'exposition s'appuie sur le comparatisme technique pour présenter les innovations issues du travail des architectes au cours de la Seconde Guerre mondiale, elle expose aussi en partie l'importante recherche $^{6}$ qui a servi à la constituer. Dans cette production dense, la méthode d'appréhension allusive préconisée par le commissaire ne s'éloigne pas de l'information; le risque de mésinterprétation est par conséquent réduit au minimum. La présentation par thèmes permet une concentration des contenus qui favorise, pour les visiteurs plus avertis, la construction de liens avec divers cadres théoriques. Enfin, le parcours de l'exposition se double d'un message réflexif qui évalue l'éthique de la pratique architecturale. De toute évidence, la portée de cette histoire possède un poids duquel il est encore aujourd'hui impossible de s'abstraire, puisqu'elle a manifestement concouru à une réorientation politique de l'économie mondiale avec laquelle il faut encore composer.

Le centre d'étude du Centre Canadien d'Architecture a inauguré l'automne dernier deux nouveaux programmes de Possibilités en muséologie visant à approfondir la recherche et l'expérimentation au sein de la pratique muséologique pour répondre aux besoins particuliers que requiert l'espace d'exposition voué à l'architecture. Le premier, Programme pour jeune commissaire, offre une résidence d'une durée allant jusqu'à trois mois qui a pour but la présentation et la réalisation d'un projet s'inscrivant dans le contexte du débat contemporain en architecture, en urbanisme et en architecture du paysage. Le second, Programme de stage en muséologie de Power Corporation du Canada, invite les jeunes étudiants et diplômés des écoles d'architecture et de design à collaborer avec le CCA pour un stage variant de six à douze mois leur permettant de se familiariser avec la collection et les programmes de conservation, d'éducation et d'exposition.

Les premiers lauréats de ce concours ouvert à l'international ont été depuis peu désignés ; l'imposante réception de candidatures a confirmé la seconde édition de cet appel, prévue pour 2012. L'information à ce sujet est disponible en ligne: <http:// www.cca.qc.ca/fr/le-cca-propose/1377-possibilites-en-museologie-2011>.

6

L'ouvrage accompagnant l'exposition en fait foi. 